\title{
Continuously Sheared Granular Matter Reproduces in Detail Seismicity Laws
}

\author{
S. Lherminier, ${ }^{1}$ R. Planet, ${ }^{1,}$ 用V. Levy dit Vehel,,${ }^{1}$ G. Simon, ${ }^{1}$ L. Vanel,,${ }^{1}$ K. J. Måløy, ${ }^{2}$ and O. Ramos ${ }^{1}, \dagger$ \\ ${ }^{1}$ Institut Lumière Matière, UMR5306 Université Lyon 1-CNRS, Université de Lyon 69622 Villeurbanne, France. \\ ${ }^{2}$ PoreLab, The Njord Centre, Department of Physics, University of Oslo, P. O. Box 1048, 0316 Oslo, Norway.
}

(Dated: May 2, 2019)

\begin{abstract}
We introduce a shear experiment that quantitatively reproduces the main laws of seismicity. By continuously and slowly shearing a compressed monolayer of disks in a ring-like geometry, our system delivers events of frictional failures with energies following a Gutenberg-Richter law. Moreover foreshocks and aftershocks are described by Omori laws and inter-event times also follow exactly the same distribution as real earthquakes, showing the existence of memory of past events. Other features of real earthquakes qualitatively reproduced in our system are both the existence of a quiescence preceding some mainshocks, as well as magnitude correlations linked to large quakes. The key ingredient of the dynamics is the nature of the force network, governing the distribution of frictional thresholds.
\end{abstract}

For more than a century, fracture and stick-slip frictional sliding have tried to explain the behavior of earthquakes. Brittle fracture induced by shear [1] was the most accepted model until the sixties. However, a more precise analysis of the radiated waves [2], the low amount of stress released by an earthquake in relation to the available one, the high energies needed to shear over a fractured surface, and over all, the lack of healing required to generate a second earthquake at the same location and close in time to the first one, set stick-slip sliding mechanisms as a more plausible explanation of earthquakes [3]. Despite these facts, the subcritical fracture of heterogeneous materials shows naturally a jerky behavior that seems closer to earthquake statistics than frictional sliding, which commonly displays a quasi-periodic stick-slip dynamics. Indeed, several fracture experiments [4-8] and numerical models [9, 10] have reported statistics of events following powerlaw distributions of sizes that have been compared to the the Gutenberg-Richter law [11]. The existence of aftershocks that follow the Omori law [12] are also common in fracture experiments [5-8].

Concerning stick-slip frictional sliding, different laboratory experiments have analyzed the sliding dynamics between two solid blocks. From a physical perspective, studies on acrylic blocks have focused on the complex evolution of the frictional strength during the slipping process, describing the behavior as a dynamic fracture problem [13, 14]. Recent friction experiments on rocks have reported results on supershear ruptures [15] and precursory activity prior to stick-slip instabilities [16]. Precursory activity to stick-slip instabilities has been also reported in experiments shearing a layer of granular material [17]. Other relevant results on similar experimental systems include remote triggering [18], and the controlled slowing down of the dynamics [19]. However, one common limitation of many of those laboratory experiments is the fact that they show a main dynamics consisting in a quasi-periodic stick-slip behavior with a narrow distribution of sizes, which

\footnotetext{
* Present address: Departament de Física de la Matèria Condensada, Universitat de Barcelona, Martí i Franquès 1, E-08028 Barcelona, Spain and Universitat de Barcelona, Institute of Complex Systems, Martí i Franquès 1, E-08028 Barcelona, Spain.

† osvanny.ramos@univ-lyon1.fr
}

do not correspond with the complex dynamics of real earthquakes described by the laws of seismicity. Other experimental systems have also sheared a granular layer, aiming at mimicking the intermittent behavior of a tectonic fault [20, 21]. Nevertheless it has been difficult to obtain a distribution of events that resembles the Gutenberg-Richter law [11] due to
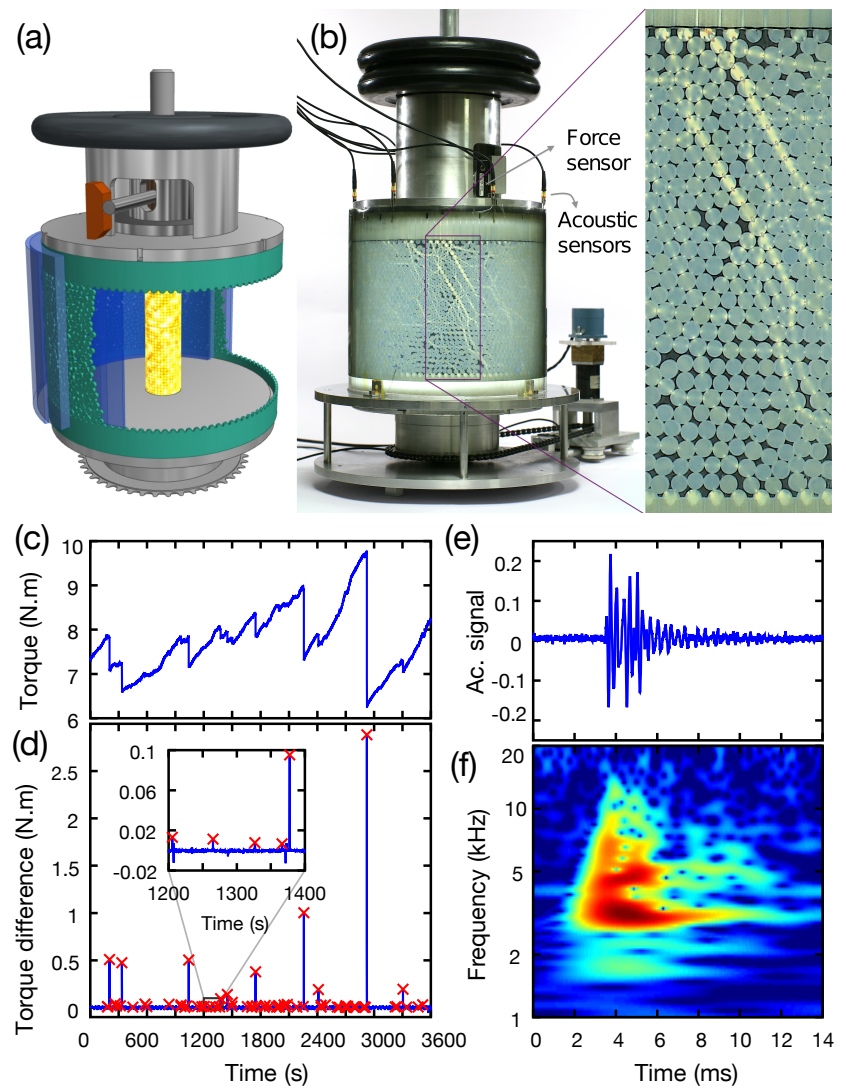

Figure 1. (color online) Setup and raw data analysis. (a) Sketch of the setup. (b) Photograph of the setup, displaying the mechanical and acoustic sensors. Inset: force chains in the granular layer observed thanks to photoelasticity. (c, d) Respectively torque signal and torque difference on $0.1 \mathrm{~s}$ intervals on a 1 hour window. Detected torque drops have been highlighted by $\times$. (e) Typical acoustic event. (f) Result of the Discrete Wavelet Transform (DWT) on the acoustic event, resulting in a power spectrogram, with a color proportional to the logarithm of the energy value. 
insufficient statistical sets of data.

Here we introduce a shear experiment capable of quantitatively reproducing the main statistical laws describing seismicity (Gutenberg- Richter law [11], Omori law [12], distribution of inter-event times [22]), as well as sharing many other qualitatively similarities with earthquake dynamics. As far as we know, it is the first time that such a quantitative agreement concerning simultaneously three main laws of seismicity is reported in a shear experiment (please, see more about the need of quantitative analogies at the Supp. Mat. [23], which includes Refs. [24-33].) Its circular geometry allows the system to reach large absolute strains (above 100\%), and more important to run continuously, capturing the considerable statistics required to analyze the dynamics of very large events, which rarely take place.

Experimental System. We study a 2D cylindrical pile confined in between two concentric fixed acrylic cylinders, and bounded by two rough circular rings (Fig. 1a, b, Movie S1), with a dead load placed over the top ring and compressing the granular pile. The top ring is free to move vertically but not to rotate, while the bottom one is slowly rotated with a period of $18.3 \overline{3}$ hours, quasi-statically shearing the granular pile with a linear velocity of $48.84 \mathrm{~mm} /$ hour (approximately 12,600 times faster than the San Andreas fault, with an average slip rate of $33.9 \mathrm{~mm} /$ year [34]). Thanks to a lever and a force sensor, we measure the torque $\Gamma(t)$ applied by the granular pile on the top ring. Six piezoelectric pinducers are inserted regularly in the top ring and simultaneously record acoustic emissions (AE). Both measures are done at a rate of 100,000 samples per second. The system is left to evolve for typical times of 24 hours.

During the shear, $\Gamma(t)$ shows an irregular stick-slip like behavior compatible with earthquakes dynamics, consisting in a continuous loading interrupted by intermittent drops with a large distribution of sizes (Fig. 1k). The detection of the torque drops (Fig. 1 $\mathrm{d}$ ) is performed by applying a threshold to mechanical energy variations $\Delta\left(\Gamma^{2}(t)\right)$ (see Appendix 1 for details). Acoustic events are linked to local releases of energy taking place at the two-dimensional interfaces between grains [35]. The analysis of acoustic recordings (Fig. 11) is based on a Discrete Wavelet Transform (DWT) [36] resulting in a time-frequency energy distribution map (Fig. 1f), that is then processed to detect peaks, corresponding to the energy of the events (details in the Supp. Mat.). The high number of events detected with both methods, respectively around 2,000 torque drops and more than 1.8 million acoustic emissions for a 24 hours experiment, allows to compute precise statistical characteristics of the system's behavior and to compare it with the dynamics of earthquakes.

Reproducing main seismicity laws. Gutenberg-Richter: On Fig. $2 \mathrm{a}$ we present the probability distribution of both acoustic $E_{\mathrm{ac}}$ and mechanical $E_{\mathrm{m}}$ energies of detected events, on logarithmic intervals. The alignment of both types of energy on the abscissa axis has been obtained from Fig. S1, where synchronous corresponding mechanical and acoustic events are represented. For one given mechanical energy, the associated acoustic one is obtained as the median energy of corresponding acoustic emissions.

The two probability density functions behave like power
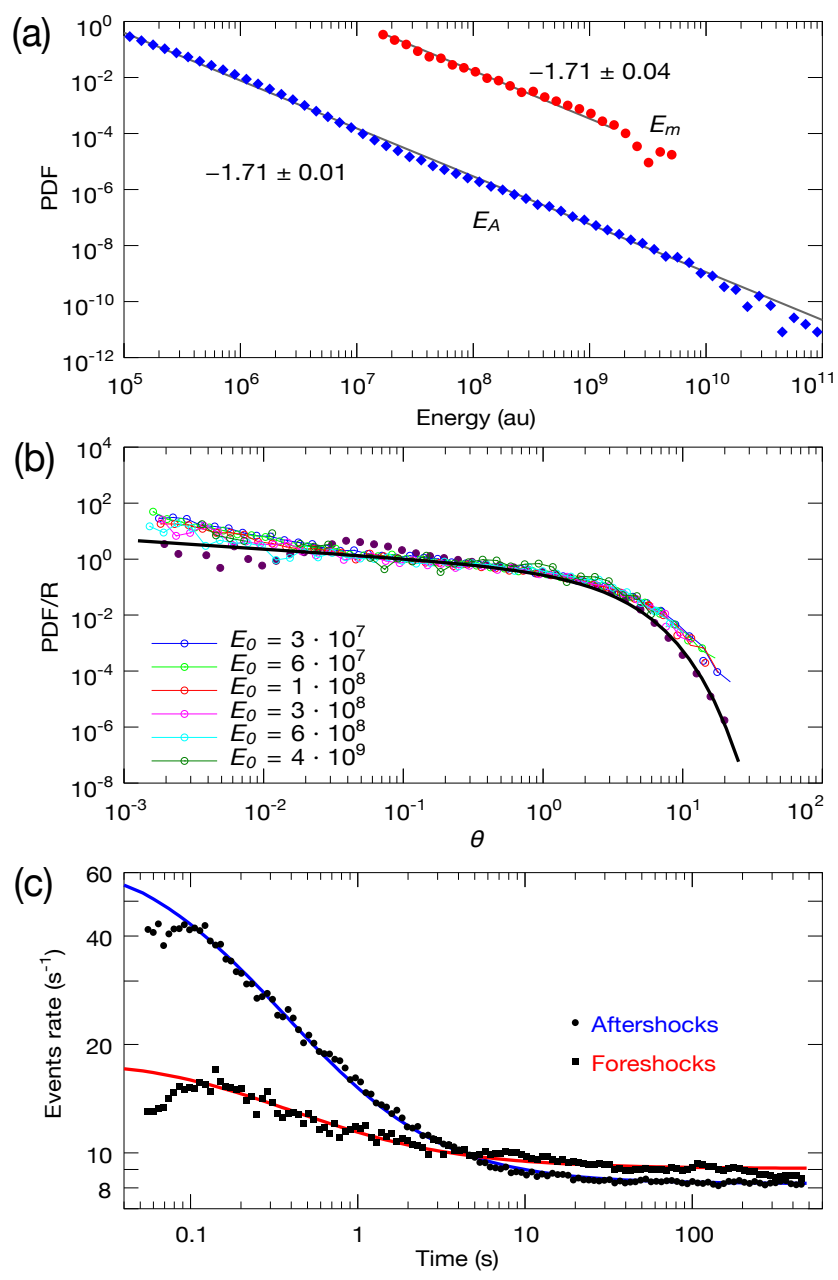

Figure 2. (color online) Quantitative reproduction of main seismicity laws. (a) Probability distribution of energies, either acoustic $E_{a}$ or mechanical $E_{m}$ for a 24-hours experiment. Both distributions follow Gutenberg-Richter like laws $P(E) \sim E^{-\beta}$ with an exponent $\beta=1.71$ (solid lines). (b) normalized probability distribution of $\theta=\tau_{E \geqslant E_{0}} / \tau_{E \geqslant E_{0}}^{*}$. Solid symbols: considering all the events. It follows the universal function $f(\theta) \sim \theta^{-0.3} \exp (-\theta / 1.5)$ (solid line). Open symbols: considering different threshold values $E_{0}$. (c) Average acoustic emissions rates respectively before (squares) and after (circles) a mainshock. They follow Omori laws, respectively $n\left(t_{-}\right)=9.05+2.78 /\left(0.22+t_{-}\right)^{0.8}$ for foreshocks and $n\left(t_{+}\right)=8.22+7.81 /\left(0.12+t_{+}\right)$for aftershocks.

laws $P(E) \sim E^{-\beta}$ with exponents $\beta_{\mathrm{m}}=1.71 \pm 0.04$ and $\beta_{\mathrm{ac}}=1.71 \pm 0.01$ for mechanical and acoustics energies respectively, obtained by a maximum likelihood method [37, 38]. The AE energies spread over six decades while the mechanical energies cover only three decades, showing the better sensitivity of the acoustic detection. This power-law behavior is to be compared with the Gutenberg-Richter law [39, 40] which states that the PDF of radiated energies of globally measured real earthquakes follows a power-law with an exponent $\beta=5 / 3=1.67$.

Inter-event times: By defining thresholds in energy $E_{0}$ (or in magnitude $M=2 / 3 \log E-2.9$, which is more used in earthquakes studies [40]) we can analyze the inter-event time between two consecutive events $\tau_{E \geqslant E_{0}}\left(\right.$ or $\tau_{M \geqslant M_{0}}$ ). In 
nature, the rate of seismicity $R_{M \geqslant M_{0}}$, defined as the number of earthquakes larger that a given magnitude $M_{0}$ per unit time varies depending on the region (eg. $R_{M \geqslant 2} \sim 15,000$ earthquakes/year for California [41]). However, the distribution of $\theta=\tau_{M \geqslant M_{0}} / \tau_{M \geqslant M_{0}}^{*}$ is a universal function following $f(\theta) \sim \theta^{-0.3} \exp (-\theta / 1.5)$ for all seismic zones and magnitude thresholds [22], where $\tau_{M \geqslant M_{0}}^{*}$ is a characteristic time defined as the inverse of the rate of seismicity $\tau_{M \geqslant M_{0}}^{*}=1 / R_{M \geqslant M_{0}}$. Although the rate of all our AE events corresponds to $R=17.35$ events/s (more than 36,000 times higher than Californian earthquakes), which gives a characteristic time $\tau^{*}=57.64 \mathrm{~ms}$, the $\theta$ distribution of our AE events follows quantitatively the same universal function (Fig. 2p).

The universal function tells us that the system "remembers" the events of energy $E \geqslant E_{0}$ during a time corresponding to their characteristic time $1 / R_{E} \geqslant E_{0}$, where the distribution is a power law. However, this implied memory, as in the case of earthquakes, is quite weak and carries no significant predictive capabilities [42]. For longer inter-event times the exponential tail of the distributions indicates that the events are independent.

For large threshold values $\left(E_{0}\right)$, the distributions deviate from the universal law both for small and large $\theta$ values (Fig. 2p). The increase of short inter-event times is a direct consequence of the increase of the activity associated to aftershocks and foreshocks in the dynamics, which is also a feature of real earthquake data [22]. The increase of long interevent times is linked to insufficient statistics. In order to verify that, we have analyzed the distribution of inter-event times $\tau_{\ell}$ for large events only $\left(E_{0}=10^{8}\right)$ (Fig. S2a). It presents two regimes: a clustering of events for short inter-event times $\left(\tau_{\ell}<\tau^{*}\right)$, and an exponential decay $D\left(\tau_{\ell}\right) \sim \exp \left(-\tau_{\ell} / \tau_{c}\right)$ indicating that large events separated by long inter-event times $\left(\tau_{\ell}>\tau^{*}\right)$ are independent and follow a Poissonian process. When we increase the energy threshold $E_{0}$ defining the large events (notice that $R_{E \geqslant E_{0}} \sim E_{0}^{1-\beta}$ ), we expect a linear relation between $\tau_{c}$ and $\tau^{*}$. However, we find that the increase of $\tau_{c}$ is slower than linear, and the best fit shows a power law with an exponent $0.86 \pm 0.01$ (Fig. S2b). This deviation may be caused by a lack of statistics concerning very large events and eventually may be used as an analytical tool to estimate biases in the obtained results due to insufficient statistical sets of data.

Omori: By defining the large acoustic emissions $\left(E_{\mathrm{ac}} \geqslant\right.$ $10^{8}$ ) as mainshocks, corresponding to about 4,500 events, we are able to reveal the existence of foreshocks and aftershocks following Omori laws as for real earthquakes [12]: $n(t)=A /(c+t)^{p}+B$, where $A / c^{p}$ gives the rate increase associated to the mainshock, $B$ the background rate of the earthquakes, $c$ the time offset (positive and close to zero) [43] and $p$ the Omori exponent, around 1. On Fig. 2k we show the average of the AE rate of foreshocks and aftershocks around the mainshocks (where time of foreshocks is $t_{-}=t_{m}-t$ and time of aftershocks $t_{+}=t-t_{m}$, with $t_{m}$ the time of the mainshock). The foreshocks rate follows an Omori-like increase $n\left(t_{-}\right)=9.05 \mathrm{~s}^{-1}+2.78 /\left(0.22 \mathrm{~s}+t_{-}\right)^{0.8}$ with a reduction of the activity in the last $0.1 \mathrm{~s}$ preceding the mainshock. Just after the mainshock, the aftershocks rate presents first a plateau, as- sociated in real earthquakes to catalogues incompleteness (due to large quakes masking smaller ones), followed by a powerlaw decrease as $n\left(t_{+}\right)=8.22 \mathrm{~s}^{-1}+7.81 /\left(0.12 \mathrm{~s}+t_{+}\right)^{1.0}$. When choosing larger threshold values defining the mainshocks (Fig. S2b) the number of events reduces; but the behavior is qualitatively the same.

Around mainshocks. Beyond analyzing the main laws of seismicity, we can also study the system's behavior around mainshocks. We divide the time axis into windows of $200 \mathrm{~ms}$ duration, from 15 seconds before to 10 seconds after a mainshock, allowing computing a local probability distribution of acoustic energies found at a given time of any of the large emission. We find power law distributions, with a variable exponent represented on Fig. 3. Far from a mainshock, we find a constant value close to 1.85 , slightly bigger than the global $\beta$-value of 1.71. This difference is caused by the selection of time windows without extreme events since they are used as reference mainshocks. For about 4 seconds before the mainshock, the exponent shows a continuous but slight decrease that accelerates in the last second to reach a value of about 1.6 just before the mainshock. The first calculated $\beta$ value after the mainshock reaches 1.4 and then it jumps again to 1.85 . These low $\beta$-values indicate an abundance of highenergy events, which are independent of the increase of the rate of the events (foreshocks and aftershocks). This sub-
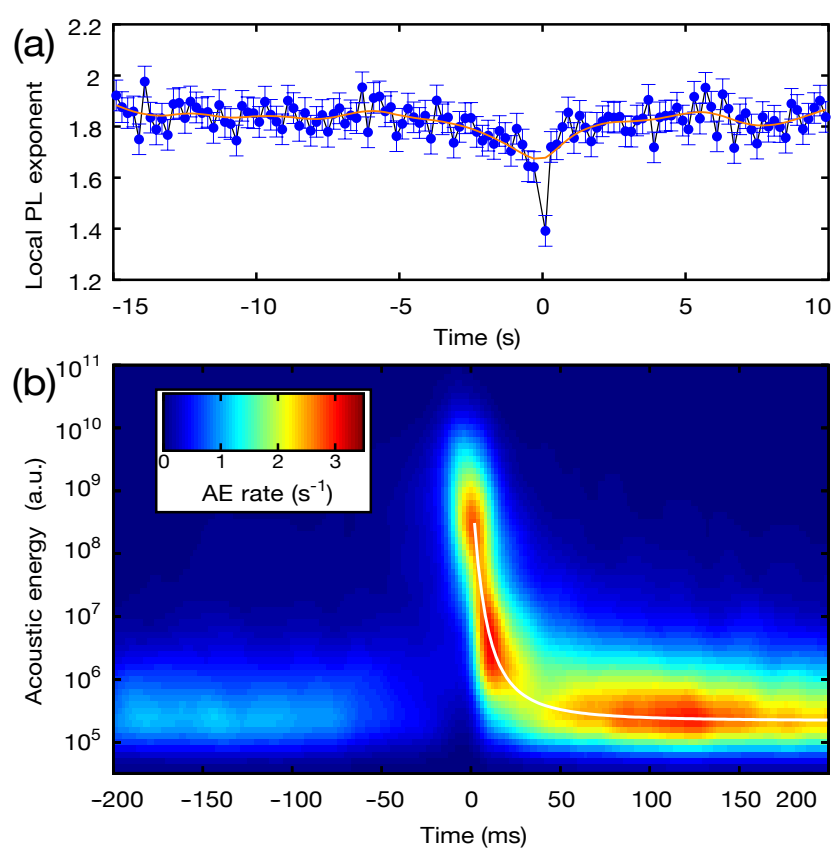

Figure 3. (color online) Around mainshocks. (a) Local power-law exponent computed on all acoustic foreshocks or aftershocks detected in fixed $200 \mathrm{~ms}$-windows centered at a given time from the mainshock. (b) Histogram of acoustic emissions energies on $2 \mathrm{~ms}$ intervals during $\pm 200 \mathrm{~ms}$ around a large acoustic emission. The AE rate is displayed as shades of color, with a corresponding numerical value averaged over the 4,500 mainshocks. We observe an abundance of high amplitudes right after the mainshock, with a decrease of the logarithm of the most probable acoustic energy value as a power-law of time (with an offset) $5.33+[60.34 /(t+44.91)]^{4.82}$, also displayed as a white solid line. 
ject is a source of controversy in the Statistical Seismology community [44, 45]. Indeed, the analysis of local and relative fast variations of the $\beta$-value in real earthquakes is well documented, often associated to correlation between magnitudes [45], but always affected by both the intrinsic lack of statistics and the incompleteness of the catalogues [44]. As our experiments rely on single measurements captured during a relative short period (one or a few days), the reported results are less affected from catalogue incompleteness than real earthquake data, making our statistical results very reliable.

We can focus on the two closest data points around the mainshock of Fig. 33. The corresponding 2D histogram (Fig. 3b) shows that high-energy events cluster just after the mainshock. They have been detected -thanks to the wavelet analysis- inside the acoustic envelopes of the mainshocks, which can last for up to $30 \mathrm{~ms}$. As large events correspond to rearrangements involving a large number of grains, it is expected to detect secondary peaks associated to the mainshocks. A priori the Omori law may not explain the statistics during these dynamic rearrangement, thus magnitude correlations seems the appropriated term to refer to this clustering of high energy events. Indeed, the activity seems to restart progressively from $40 \mathrm{~ms}$ having a maximum around 120 $\mathrm{ms}$. These results, coherent with the aftershocks displayed on Fig. 2 k, indicate that the flat part of the Omori law between 50 ms and 120 is not provoked by missing small events masked by previous large ones. We can also notice a clear quiescence in the $60 \mathrm{~ms}$ interval preceding the mainshock, also coherent with the decrease of the foreshock activity close to the mainshocks (Fig. 22), a phenomenon that has been often reported prior to some very large earthquakes [46]. Longterm decrease of $\beta$-values has also been reported preceding some very large earthquakes [47], and also in controlled experiments [48] and simulations [9].

Discussions. A very large distribution of thresholds, required to achieve an earthquake-like dynamics, is directly related to the heterogeneous character of the system. In subcritical fracture experiments like those cited in the introduction [4]-8], the heterogeneity is provided by a structural disorder, and the competition between the advancing crack and the fracture thresholds may result in a Gutenberg-Richter-like distribution of event sizes. However, most shear experiments present a main dynamics composed of quasi-periodic stickslip events with a narrow distribution of sizes, which may be a consequence of a lack of disorder. In the case of similar experiments shearing a granular layer [17--19], a very high number of particles and three-dimensional force chains may be responsible for an "averaging" effect that reduces the heterogeneity of the system, resulting in a regular stick-slip dynamics similar to the one obtained in solid flat interfaces.

In our system, the granular force network [49-51] provides an emergent and evolving heterogeneity in terms of energy thresholds that is the key ingredient of the dynamics, and it is responsible for a distribution of events that resembles the Gutenberg-Richter law [11, 40]. The structure, dynamics and sizes of these heterogeneities in an actual fault remain as open questions. However, the two-dimensional nature of both our system, and the Hertzian and frictional inter-grain interfaces, and also the low dimensionality of the force network (which may depend on the pressure between the plates [52]) may serve as hints to eventually find them (please, see more details about the insights into real earthquakes in the Supp. Mat.). Our system is able to reproduce quantitatively the main statistical laws of seismicity, which indicates that both earthquakes and our experiment are governed by a similar physics, and opens a new pathway to the investigation of earthquake-like dynamics at a laboratory scale.

\section{ACKNOWLEDGMENTS}

We thank E. Altshuler, T. Bodin, T. Hatano, C. Lasserre C. Marone, and M. Métois for very useful discussions. We acknowledge support from the AXA Research Fund. O. R. acknowledges support from the Chaire-CNRS program and the University of Tokyo for the invited researcher position at the Earthquake Research Institute. This work was partly supported by the Research Council of Norway through its centers of Excellence funding scheme, project number 262644.
[1] H. F. Reid, "The elastic-rebound theory of earthquakes," Univ. of California Publications, Bulletin of the Department of Geology 6, 19 (1911).

[2] H. Benioff, "Earthquake source mechanisms," Science 143, 1399 (1964).

[3] W. F. Brace and J. D. Byerlee, "Stick-slip as a mechanism for earthquakes," Science 153, 990-992 (1966)

[4] C. H. Scholz, "The frequency-magnitude relation of microfracturing in rock and its relation to earthquakes," Bulletin of the seismological society of America 58, 399-415 (1968).

[5] Knut Jørgen Måløy, Stéphane Santucci, Jean Schmittbuhl, and Renaud Toussaint, "Local waiting time fluctuations along a randomly pinned crack front,' Phys. Rev. Lett. 96, 045501 (2006)

[6] Jordi Baró, Álvaro Corral, Xavier Illa, Antoni Planes, Ekhard KH Salje, Wilfried Schranz, Daniel E Soto-Parra, and Eduard Vives, "Statistical similarity between the compression of a porous material and earthquakes," Phys. Rev. Lett. 110, 088702 (2013).

[7] M. Stojanova, S. Santucci, L. Vanel, and O. Ramos, "High frequency monitoring reveals aftershocks in subcritical crack growth,' Phys. Rev. Lett. 112, 115502 (2014).

[8] J. Barés, A. Dubois, L. Hattali, D. Dalmas, and D. Bonamy, "Aftershock sequences and seismic-like organization of acoustic events produced by a single propagating crack," Nature Communications 9, 1253 (2018).

[9] Ferenc Kun, Imre Varga, Sabine Lennartz-Sassinek, and Ian G. Main, "Approach to failure in porous granular materials under compression,” Phys. Rev. E 88, 062207 (2013)

[10] Ferenc Kun, Imre Varga, Sabine Lennartz-Sassinek, and Ian G. Main, "Rupture cascades in a discrete element model of a porous sedimentary rock,' Phys. Rev. Lett. 112, 065501 (2014)

[11] Beno Gutenberg and Charles Francis Richter, "Magnitude and 
energy of earthquakes," Ann. Geophys. 9, 1-15 (1956).

[12] F. Omori, "On the aftershocks of earthquakes," J. Coll. Sci. Imp. Univ. Tokyo 7, 111-200 (1894).

[13] Shmuel M. Rubinstein, Gil Cohen, and Jay Fineberg, "Detachment fronts and the onset of dynamic friction," Nature 430, 1005-1009 (2004).

[14] Oded Ben-David, Shmuel M. Rubinstein, and Jay Fineberg, "Slip-stick and the evolution of frictional strength," Nature 463, 76-79 (2010)

[15] F. X. Passelègue, S. Schubnel, A. Nielsen, H. S. Bhat, and R. Madariaga, "From sub-rayleigh to supershear ruptures during stick-slip experiments on crustal rocks," Science 340, 1208 (2013).

[16] S. Passelègue, F. X. Latour, S. Schubnel, A. Nielsen, H. S. Bhat, and R. Madariaga, "Influence of fault strength on precursory processes during laboratory earthquakes," in Fault Zone Dynamic Processes: Evolution of Fault Properties During Seismic Rupture, Geophysical Monograph 227, edited by M Thomas, T. M. Mitchell, and Bhat H. S. (Washington, DC: American Geophysical Union, 2016) pp. 229-242.

[17] J. Rivière, Z. Lv, P.A. Johnson, and C. Marone, "Evolution of b-value during the seismic cycle: Insights from laboratory experiments on simulated faults," Earth and Planetary Science Letters 482, 407 - 413 (2018).

[18] Paul A Johnson, Heather Savage, Matt Knuth, Joan Gomberg, and Chris Marone, "Effects of acoustic waves on stick-slip in granular media and implications for earthquakes," Nature 451, 57-60 (2008).

[19] M. M. Scuderi, C. Marone, E. Tinti, G. Di Stefano, and C. Collettini, "Precursory changes in seismic velocity for the spectrum of earthquake failure modes," Nature Geoscience 9, 695 (2016).

[20] Karen E Daniels and Nicholas W Hayman, "Force chains in seismogenic faults visualized with photoelastic granular shear experiments," J. Geophys. Res. Solid Earth 113 (2008).

[21] David M Walker, Antoinette Tordesillas, Michael Small, Robert P Behringer, and K Tse Chi, "A complex systems analysis of stick-slip dynamics of a laboratory fault," Chaos 24, 013132 (2014).

[22] Alvaro Corral, "Long-term clustering, scaling, and universality in the temporal occurrence of earthquakes," Phys. Rev. Lett. 92, 108501 (2004).

[23] Supplemental Material [url].

[24] David L. Henann and Ken Kamrin, "A predictive, sizedependent continuum model for dense granular flows," Proceedings of the National Academy of Sciences 110, 6730-6735 (2013).

[25] O. Kuwano, R. Ando, and T. Hatano, "Granular friction in a wide range of shear rates," AIP Conf. Proc. 1542, 32-37 (2013).

[26] H. E. Stanley, Introduction to Phase Transitions and Critical Phenomena (Oxford Univ. Press, New York, 1987).

[27] T. Yamashita and A. Tsutsumi, Involvement of fluids in earthquake ruptures: Field/Experimental data and modeling (Springer Japan, 2018).

[28] Ian Main, "Statistical physics, seismogenesis, and seismic hazard," Reviews of Geophysics 34, 433-462 (1996)

[29] Takahiro Hatano, Clément Narteau, and Peter Shebalin, "Common dependence on stress for the statistics of granular avalanches and earthquakes," Sci Rep 5, 12280 (2015)

[30] USGS site: http://earthquake.usgs.gov/earthquakes/search/.

[31] Alessandro Vespignani and Stefano Zapperi, "Order parameter and scaling fields in self-organized criticality," Phys. Rev. Lett. 78, 4793-4796 (1997).

[32] Zeev Olami, Hans Jacob S. Feder, and Kim Christensen, "Selforganized criticality in a continuous, nonconservative cellular automaton modeling earthquakes,” Phys. Rev. Lett. 68, 1244 1247 (1992).

[33] Juan A Bonachela and Miguel A Muñoz, "Self-organization without conservation: true or just apparent scale-invariance?" Journal of Statistical Mechanics: Theory and Experiment 2009, P09009 (2009).

[34] Kerry E. Sieh and Richard H. Jahns, "Holocene activity of the san andreas fault at wallace creek, california," GSA Bulletin 95, 883 (1984)

[35] F. Dubourg, S. Lherminier, R. Planet, K. Rapina, F. Bunel, L. Vanel, and O. Ramos, "The sound of avalanches: from a global to a local perspective," EPJ Web Conf. 140, 03015 (2017)

[36] C. K. Chui, An Introduction to Wavelets (Academic Press, San Diego, 1992).

[37] Aaron Clauset, Cosma Rohilla Shalizi, and Mark EJ Newman, "Power-law distributions in empirical data," SIAM rev. 51, 661-703 (2009).

[38] Jeff Alstott, Ed Bullmore, and Dietmar Plenz, "powerlaw: A python package for analysis of heavy-tailed distributions," PLOS ONE 9, 1-11 (2014).

[39] Thomas C Hanks and Hiroo Kanamori, "A moment magnitude scale,” J. Geophys. Res. B: Solid Earth 84, 2348-2350 (1979).

[40] George L. Choy and John L. Boatwright, "Global patterns of radiated seismic energy and apparent stress," J. Geophys. Res. Solid Earth 100, 18205-18228 (1995)

[41] Karen R. Felzer, Calculating California Seismicity Rates, Tech. Rep. (2008) u.S. Geological Survey Open-File Report.

[42] Sarah Touati, Mark Naylor, and Ian G. Main, "Origin and nonuniversality of the earthquake interevent time distribution," Phys. Rev. Lett. 102, 168501 (2009)

[43] Bogdan Enescu, Jim Mori, and Masatoshi Miyazawa, "Quantifying early aftershock activity of the 2004 mid-niigata prefecture earthquake (mw 6.6), J. Geophys. Res. 112, B04310 (2007).

[44] Jörn Davidsen and Adam Green, "Are earthquake magnitudes clustered?" Phys. Rev. Lett. 106, 108502 (2011).

[45] E. Lippiello, C. Godano, and L. de Arcangelis, "The earthquake magnitude is influenced by previous seismicity," Geophys. Res. Lett. 39, L05309 (2012).

[46] H. Kanamori, "The nature of seismicity patterns before major earthquakes," in Earthquake Prediction, an International Review, edited by D.W.. Simpson and P.G. Richards (Maurice Ewing Series, Vol. IV, Am. Geophys. Union, Washington D.C, 1981) pp. 1-19.

[47] K. Z. Nanjo, N. Hirata, K. Obara, and K. Kasahara, "Decadescale decrease in b value prior to the m9-class 2011 tohoku and 2004 sumatra quakes," Geophys. Res. Lett. 39, L20304 (2012)

[48] P. R. Sammonds, P. G. Meredith, and I. G. Main, "Role of pore fluids in the generation of seismic precursors to shear fracture," Nature 359, 228-230 (1992).

[49] Brian Miller, Corey O'Hern, and R. P. Behringer, "Stress fluctuations for continuously sheared granular materials," Phys. Rev. Lett. 77, 3110-3113 (1996)

[50] Daniel Howell, R. P. Behringer, and Christian Veje, "Stress fluctuations in a $2 \mathrm{~d}$ granular couette experiment: a continuous transition,” Phys. Rev. Lett. 82, 5241 (1999).

[51] T. S. Majmudar and R. P. Behringer, "Contact force measurements and stress-induced anisotropy in granular materials," Nature 435, 1079-1082 (2005).

[52] S Lherminier, R Planet, G Simon, L Vanel, and O Ramos, "Revealing the structure of a granular medium through ballistic sound propagation,” Phys. Rev. Lett. 113, 098001 (2014). 